\title{
STUDY OF MAGNETIC FIELD DEPENDENT VISCOSITY ON A SORET DRIVEN FERROTHERMOHALINE CONVECTION IN A ROTATING POROUS MEDIUM
}

\author{
R. HEMALATHA \\ Mahatma Gandhi Government Arts College \\ Mahe - 673311, INDIA \\ E-mail: hemalatha53@yahoo.co.in
}

\begin{abstract}
The effect of a magnetic field dependent viscosity on a Soret driven ferro thermohaline convection in a rotating porous medium has been investigated using the linear stability analysis. The normal mode technique is applied. A wide range of values of the Soret parameter, magnetization parameter, the magnetic field dependent viscosity, Taylor number and the permeability of porous medium have been considered. A Brinkman model is used. Both stationary and oscillatory instabilities have been obtained. It is found that the system stabilizes only through oscillatory mode of instability. It is found that the magnetization parameter and the permeability of the porous medium destabilize the system and the Soret parameter, the magnetic field dependent viscosity and the Taylor number tend to stabilize the system. The results are presented numerically and graphically.
\end{abstract}

Key words: Soret parameter, field dependent viscosity, oscillatory instability, linear stability, Taylor number, permeability of porous medium.

\section{Introduction}

Ferrofluids are single-magnetic-domain, two-phase three component fluids (Rosensweig, 1985), where the core represents the single domain, the core and carrier fluid represent the two phases, the surfactant and carrier fluids represent the three components. A distinguishing feature of a ferrofluid is its promising applications in various disciplines. Some practical applications of ferrofluids are dynamic sealing, heat dissipation, damping and doping of technological materials.

Ferroconvection studies were initiated by Cowley and Rosensweig (1947) followed by Finlayson (1970) and Lalas and Carmi (1971). Finlayson (1970) studied the convective instability of a single component ferrofluid heated from below in the presence of a vertical uniform magnetic fluid and explained the concept of thermo mechanical interaction in a ferrofluid. Bernard convection (Chandrasekhar, 1961) in magnetic fluids has been analyzed by Schechter and Velarde (1974) and Schwab et al. (1993).

Double diffusive convection is of great importance in various fields such as high quality crystal production, oceanography, production of pure medicine, solidification of molten alloys, geothermally heated lakes and magmas. Double diffusive convection occurs when the above system is heated up. In this convection, mass transfer is included and the density variation is caused by two different components which have different rates of diffusion (Huppert and Turner 1981; Turner, 1974). Vaidyanathan et al. (1995; 1997) investigated the ferrothermohaline convection in the presence and absence of a porous medium. The solute is ferromagnetic, which modifies the magnetic field established as a perturbation. They obtained the conditions for the onset of thermal stability through stationary and oscillatory modes.

When a salty fluid gets heated up two types of interdiffusive phenomena occur. They are known as (i) the Soret effect, (ii) Dufour effect. Thermodiffusion, also called the Soret effect, is characterized by the Soret coefficient. Thermodiffusion in a ferrofluid in the presence of a magnetic field was investigated by Voelker and Odenbach (2005). 
Hurle and Jakeman (1971) analyzed the Soret-driven thermosolutal convection. They experimentally analyzed the non-linear stability of double diffusive phenomena. Self-oscillatory convection caused by the Soret effect was studied by Shliomis and Souhar (2000). They analyzed the influence due to magnetophoreis and the Soret effect in the convective instability of magnetized ferrofluids. By considering the magnetic fluid as a binary mixture, thermodiffusive problems in magnetic fluids in the presence of magnetic fields were analyzed by Lange (2004). Shevtsova et al. (2006) carried out a study on the onset of convection in Soret driven instability. Soret - driven convection in a horizontal porous layer was analytically and numerically studied by Charier - Mojtabi et al. (2007). They found that for a cell heated from below the monocellular flow loses stability and when it is heated from above it remains stable.

Soret driven ferrothermohaline convection in the presence and absence of a porous medium was investigated by Vaidyanathan et al. (2005) and Sekar et al. (2006). They also analyzed the effect of dust particles in ferrothermohaline convection due to the Soret effect (Sekar et al., 2008 and 2009) and (Hemalatha et al., 2011). A linear stability analysis on the onset of Soret driven motion in nanoparticles suspension was made by Kim (2011).

The effect of rotation on ferrothermohaline convection saturating a porous medium was analyzed by Sekar et al. (1998). A non linear stability analysis of a rotating double diffusive magnetized ferrofluid was carried out by Sunil et al. (2011). The effect of porosity on revolving ferrofluid flow with a rotating disk was analyzed by Kushal Sharma et al. (2011a).

It is interesting to study the nature of variable viscosity on fluids. Viscosity may depend on temperature (Ramanathan and Mukhil, 2006) and the magnetic field also. Thermal convection in a ferromagnetic fluid in the presence of a magnetic field dependent viscosity was investigated by many authors (Kushal Sharma et al., 2010; 2011b) and (Vaidyanathan et al., 2002a; 2002b and 2002c). The effect of a magnetic field dependent viscosity on ferroconvection and ferrothermohaline convection in the presence and absence of dust particles was studied by Sunil et al. (2005 and 2006). Nanjundappa et al. (2009) analyzed the effect of a magnetic dependent viscosity on the onset of a ferromagnetic fluid layer heated from below and cooled from above with constant heat flux. The effect of a magnetic field dependent viscosity on ferroconvection in an anisotropic porous medium in the presence of a horizontal thermal gradient was studied by Hemalatha and Sivapraba (2012).

Vaidyanathan et al. (2007) discussed the effect of Coriolis force on a Soret driven ferrothermohaline convection in a medium of sparse particle suspension. In the present work, it is intended to include the effect of a magnetic field dependent viscosity, Coriolis force and Soret effect in a ferrofluid saturating a porous medium. A linear stability analysis has been carried out. A Brinkman model is used. It is found that the system stabilizes only through oscillatory mode. The values are presented graphically.

\section{Mathematical formulation}

A horizontal layer of an incompressible Boussinesq ferromagnetic fluid of thickness ' $d$ ' in the presence of a transversely applied magnetic field heated from below and salted from below and above is considered. The temperature and salinity at the bottom and top surfaces $z= \pm d / 2$ are $T_{0} \mp \Delta T / 2$ and $S_{0} \pm$ $\Delta S / 2$ respectively. Both boundaries are taken to be free and perfect conductors of heat and solute. Considering the Soret effect on the temperature gradient the mathematical equations governing the above investigation are as follows.

The fluid is assumed to be an incompressible fluid having a variable viscosity given by

$$
\mu=\mu_{1}(1+\delta \cdot B)
$$

where $\mu_{l}$ is taken as the viscosity of the fluid when the applied magnetic field is absent. The variation in the coefficient of the magnetic field dependent viscosity $\delta$ has been taken to be isotropic $\delta_{1}=\delta_{2}=\delta_{3}$. Hence the component wise $\mu$ can be written as 


$$
\begin{aligned}
& \mu_{x}=\mu_{1}\left(1+\delta B_{1}\right), \\
& \mu_{y}=\mu_{1}\left(1+\delta B_{2}\right), \\
& \mu_{z}=\mu_{1}\left(1+\delta B_{3}\right) .
\end{aligned}
$$

The continuity equation for an incompressible Boussinesq fluid is

$$
\nabla \cdot \boldsymbol{q}=0
$$

The momentum equation as given by Finlayson (1970) is

$$
\rho_{0} \frac{D \boldsymbol{q}^{\prime}}{D t}=-\nabla p+\rho \boldsymbol{g}+\nabla \cdot(\boldsymbol{H} \boldsymbol{B})+\mu \nabla^{2} \boldsymbol{q}^{\prime}+2 \rho_{0}\left(\boldsymbol{q}^{\prime} \times \boldsymbol{\Omega}\right)-\frac{\mu}{k} \boldsymbol{q}^{\prime} .
$$

The temperature equation for an incompressible ferrofluid is

$$
\left[\rho_{0} C_{v, H}-\mu_{0} \boldsymbol{H} \cdot\left(\frac{\partial \boldsymbol{M}}{\partial T}\right)_{v, H}\right] \frac{d T}{d t}+\mu_{0} T\left(\frac{\partial \boldsymbol{M}}{\partial T}\right)_{v, H} \cdot \frac{d \boldsymbol{H}}{d t}=K_{l} \nabla^{2} T+\varphi .
$$

The mass flux equation is given by

$$
\frac{D S}{D t}=K_{S} \nabla^{2} S+\mathrm{S}_{\mathrm{T}} \nabla^{2} T
$$

where $K_{l}, K_{S}, \varphi$, and $\mathrm{S}_{\mathrm{T}}$ are the thermal conductivity, concentration diffusivity, viscous dissipation factor containing second order terms in velocity and the Soret coefficient respectively.

Using Maxwell's equations for non-conducting fluids, one can assume that the magnetization is aligned with the magnetic field and depends on the magnitude of the magnetic field, temperature and salinity, so that

$$
\boldsymbol{M}=\frac{\boldsymbol{H}}{H} M(H, T, S) .
$$

The magnetic equation of state is linearized about the magnetic field $H_{0}$, the average temperature $T_{0}$ and the average salinity $S_{0}$ to become

$$
M=M_{0}+\chi\left(H-H_{0}\right)-K\left(T-T_{0}\right)+K_{2}\left(S-S_{0}\right)
$$

where $\quad \chi=\left[\frac{\partial M}{\partial H}\right]_{H_{0, T_{0}}}, \quad K=-\left[\frac{\partial M}{\partial T}\right]_{H_{0} T_{0}}, \quad K_{2}=\left[\frac{\partial M}{\partial S}\right]_{H_{0}, S_{0}}$ are the magnetic susceptibility, the pyromagnetic coefficient and the salinity magnetic coefficient, respectively.

The density equation of state for a Boussinesq two-component fluid is

$$
\rho=\rho_{0}\left(1-\alpha_{t}\left(T-T_{0}\right)+\alpha_{s}\left(S-S_{0}\right)\right)
$$


where $\alpha_{t}$ is the thermal coefficient and $\alpha_{s}$ the solute analog of $\alpha_{t}$. The basic state is assumed to be a quiescent state and the basic state quantities are obtained by substituting the velocity of the quiescent state in the governing Eqs (2.1)-(2.5) and the solutions of Eqs (2.1)-(2.8) are obtained using the techniques of the linear stability analysis and normal mode technique.

Normal mode solution of all dynamical variables can be written as

$$
f(x, y, z, t)=f(z, t) \exp i\left(k_{x} x+k_{y} y\right)
$$

where $f(z, t)$ represents $[w(z, t), \theta(z, t), S(z, t), z(z, t), \varphi(z, t)]$.

The wave number $k_{0}$ is given by

$$
k_{0}^{2}=k_{x}^{2}+k_{y}^{2} .
$$

The modified Fourier heat conduction equation is

$$
\begin{aligned}
& {\left[\rho_{0} C_{V, H} \frac{\partial \theta}{\partial t}-\mu_{0} K T_{0} \frac{\partial}{\partial t}\left(\frac{\partial \varphi}{\partial z}\right)\right]=\left[K_{1}\left(\frac{\partial^{2}}{\partial z^{2}}-k_{0}^{2}\right) \theta+\right.} \\
& \left.+\left[\rho_{0} C \beta_{t}-\left(\frac{\mu_{0} K^{2} T_{0}^{2} \beta_{t}}{1+\chi}\right)+\left(\frac{\mu_{0} K K_{2} T_{0} \beta s}{1+\chi}\right)\right] w\right]
\end{aligned}
$$

where

$$
\rho_{0} C=\rho_{0} C_{V, H}+\rho_{0} K H_{0}
$$

The vertical component of the momentum equation can be written as

$$
\begin{aligned}
& \rho_{0} \frac{\partial}{\partial t}\left(\frac{\partial^{2}}{\partial z^{2}}-k_{0}^{2}\right) w=\left(\frac{\mu_{0} K \beta_{t}}{1+\chi}\right)\left[(1+\chi) \frac{\partial \varphi}{\partial z}-K \theta\left(1-\mathrm{S}_{\mathrm{T}}\right)\right] k_{0}^{2}-\rho_{0} g \alpha_{t} k_{0}^{2} \theta+ \\
& +\left(\frac{\mu_{0} K_{2} \beta_{S}}{1+\chi}\right)\left[(1+\chi) \frac{\partial \varphi}{\partial z}+K_{2} S\right] k_{0}^{2}+\rho_{0} g \alpha_{S} k_{0}^{2} S+ \\
& -\left(\frac{\mu_{0} K K_{2}}{1+\chi}\right)\left[\beta_{S}\left(1-\mathrm{S}_{\mathrm{T}}\right) \theta-\beta_{t} S\right] k_{0}^{2}-\mu_{1} \delta \mu_{o}(M o+H o)\left(\frac{\partial^{2}}{\partial z^{2}}-k_{0}^{2}\right) k_{0}^{2} w+ \\
& +\mu\left(\frac{\partial^{2}}{\partial z^{2}}-k_{0}^{2}\right)^{2} w-2 \rho_{0} \Omega \frac{\partial S}{\partial z}-\frac{\mu}{k}\left(\frac{\partial^{2}}{\partial z^{2}}-k_{0}^{2}\right) w .
\end{aligned}
$$

The $z$ component of the vorticity equation can easily be obtained as

$$
\rho_{0} \frac{\partial \varsigma}{\partial t}=\mu\left(\frac{\partial^{2}}{\partial z^{2}}-k_{0}^{2}\right) \varsigma+2 \rho_{0} \Omega \frac{\partial w}{\partial z}-\frac{\mu}{k} \varsigma
$$

where $\varsigma=\left(\frac{\partial v}{\partial x}-\frac{\partial u}{\partial y}\right)$, is the $z$ component of vorticity. 
The pressure term, the magnetic body force term vanish from the vorticity equation. The salinity equation is

$$
\frac{\partial S}{\partial t}+\beta_{s} w=K_{S}\left(\frac{\partial^{2}}{\partial z^{2}}-k_{0}^{2}\right) S+\mathrm{S}_{\mathrm{T}}\left(\frac{\partial^{2}}{\partial z^{2}}-k_{0}^{2}\right) \theta .
$$

The magnetic potential equation is

$$
(1+\chi) \frac{\partial^{2} \varphi}{\partial z^{2}}-\left(1+\frac{M_{0}}{H_{0}}\right) k^{2}{ }_{0} \varphi-K \frac{\partial \theta}{\partial z}+K_{2} \frac{\partial S}{\partial z}+\mathrm{S}_{\mathrm{T}} K \frac{\partial \theta}{\partial z}=0 .
$$

Following the normal mode analysis, the linearized perturbation dimensionless equations for the thermosolutal convection due to Soret effect in a ferrofluid are

$$
\begin{aligned}
& \left(\frac{\partial}{\partial t^{*}}+\frac{1}{k^{*}}\right)\left(D^{2}-a^{2}\right) w^{*}=-(\mathrm{Ta})^{1 / 2} D \varsigma^{*}+a R^{1 / 2}\left[M_{1} D \varphi^{*}-\left(1+M_{1}\left(1-\mathrm{S}_{\mathrm{T}}\right)\right) T^{*}\right]+ \\
& +M_{1} M_{5} a R^{1 / 2} D \varphi^{*}-M_{1} M_{5} a R^{1 / 2}\left(1-\mathrm{S}_{\mathrm{T}}\right) T^{*}+\left(D^{2}-a^{2}\right)^{2} w^{*}+ \\
& +a R_{s}^{1 / 2}\left(1+M_{4}+\frac{M_{4}}{M_{5}}\right) S^{*}-a^{2} \delta^{*} M_{3}\left(D^{2}-a^{2}\right) w^{*}, \\
& \left(\frac{\partial}{\partial t^{*}}+\frac{1}{k^{*}}\right) \varsigma^{*}=\left(D^{2}-a^{2}\right) \varsigma^{*}+(\mathrm{Ta})^{1 / 2} D w^{*}, \\
& \operatorname{Pr}\left[\frac{\partial T^{*}}{\partial t^{*}}-M_{2} \frac{\partial}{\partial t^{*}}\left(D \varphi^{*}\right)\right]=\left(D^{2}-a^{2}\right) T^{*}+a R^{1 / 2}\left[\left(1-M_{2}-M_{2} M_{5}\right)\right] w^{*}, \\
& \operatorname{Pr} \frac{\partial S^{*}}{\partial t^{*}}=\tau\left(D^{2}-a^{2}\right) S^{*}-a R_{S}^{1 / 2} M_{6} w^{*}+\mathrm{S}_{\mathrm{T}}\left(\frac{M_{5}}{M_{6}}\right) R_{S}^{1 / 2}\left(D^{2}-a^{2}\right) T^{*}, \\
& D^{2} \varphi^{*}-a^{2} M_{3} \varphi^{*}-\left(1-\mathrm{S}_{\mathrm{T}}\right) D T^{*}+\left(\frac{M_{5}}{M_{6}}\right)\left(\frac{R}{R_{S}}\right)^{\frac{1}{2}} D S^{*}=0
\end{aligned}
$$

where the non-dimensional variables can be written as

$$
\begin{array}{ll}
t^{*}=\frac{v t}{d^{2}}, & w^{*}=\frac{w d}{v}, \quad T^{*}=\left(\frac{K_{1} a R^{1 / 2}}{\rho_{0} C_{V, H} \beta_{t} v d^{2}}\right) \theta, \quad \varphi^{*}=\left(\frac{(1+\chi) K_{1} a R^{1 / 2}}{K \rho_{0} C_{V, H} \beta_{t} v d^{2}}\right) \varphi, \\
z^{*}=\frac{z}{d}, & a=k_{0} d, \quad D=\frac{\partial}{\partial z^{*}}, \quad S^{*}=\left(\frac{K_{S} a R_{S}^{1 / 2}}{\rho_{0} C_{V, H} \beta_{S} v d}\right) S, \quad k^{*}=\frac{k}{d^{2}}
\end{array}
$$


where the dimensionless parameters used are

$$
\begin{aligned}
& M_{1}=\frac{\mu_{0} K^{2} \beta_{t}}{(1+\chi) \rho_{0} g \alpha_{t}}, \quad M_{2}=\frac{\mu_{0} K^{2} T_{0}}{(1+\chi) \rho_{0} C_{V, H}}, \quad M_{3}=\frac{\left(1+\frac{M_{0}}{H_{0}}\right)}{(1+\chi)}, \\
& M_{4}=\frac{\mu_{0} K^{2} \beta_{S}}{(1+\chi) \rho_{0} g \alpha_{S}}, \quad \operatorname{Pr}=\frac{\mu C_{v, H}}{K_{1}}, \quad R=\frac{\rho_{0} C_{v, H} \beta_{t} \alpha_{t} g d^{4}}{v K_{1}}, \\
& M_{5}=\frac{K_{2} \beta_{S}}{K \beta_{t}}, \quad R_{S}=\frac{\rho_{0} C_{v, H} \beta_{S} \alpha_{S} g d^{4}}{v K_{S}}, \quad \tau=\rho_{0} C_{v, H}\left(\frac{K_{S}}{K_{1}}\right), \\
& M_{6}=\frac{K_{S}}{K_{1}}, \quad \quad \mathrm{Ta}=\left(\frac{2 \Omega d^{2}}{v}\right)^{2}, \quad \delta^{*}=\delta \mu_{0} H_{0}(1+\chi) M_{3}
\end{aligned}
$$

where $R, R_{S}$, Pr and $\mathrm{S}_{\mathrm{T}}$ are, respectively, the critical thermal Rayleigh number, salinity Rayleigh number, the Prandtl number, Soret coefficient and other parameters to represent non dimensional parameters used appropriately.

\section{Exact solution for free boundaries}

The boundary conditions on velocity, temperature, salinity and the magnetic potential are

$$
w^{*}=D^{2} w^{*}=T^{*}=D \varphi^{*}=S^{*}=0 \quad \text { at } \quad z^{*}= \pm \frac{1}{2} .
$$

The exact solutions satisfying Eq.(3.1) are

$$
\begin{array}{ll}
W^{*}=A_{1} e^{\sigma t^{*}} \cos \pi z^{*}, & T^{*}=B_{1} e^{\sigma t^{*}} \cos \pi z^{*}, \\
\varphi^{*}=\frac{F_{1}}{\pi} e^{\sigma t^{*}} \sin \pi z^{*}, & D \varphi^{*}=F_{1} e^{\sigma t^{*}} \cos \pi z^{*}
\end{array}
$$

where $A_{1}, B_{1}, C_{1}$, and $F_{1}$ are constants and $\sigma$ is the growth rate. In the above solution, the lowest mode of $\sin$ $(n \pi z)$, for $n=1$ is assumed as a solution. The solution can be odd or even modes compatible with boundary conditions. In the present case of choosing the reference at the centre enables one to choose the lowest even mode, namely $\cos (n \pi z)$ for all dynamical variables. Substitution of Eqs (3.2) in Eqs (2.16) - (2.20) leads to

$$
\begin{aligned}
& A_{l}\left(\left(\pi^{2}+a^{2}\right)^{2}+\left(\sigma+\frac{1}{k}\right)\left(\pi^{2}+a^{2}\right)+\frac{\mathrm{Ta} \pi^{2}}{\sigma+\frac{1}{k}+\pi^{2}+a^{2}}+a^{2} \delta M_{3}\left(\frac{1}{k}+\pi^{2}+a^{2}\right)+\right. \\
& -B_{1}\left[1+M_{1}\left(1-\mathrm{S}_{\mathrm{T}}\right)+M_{1} M_{5}\left(1-\mathrm{S}_{\mathrm{T}}\right)\right] a R^{1 / 2}+ \\
& +C_{l}\left(1+M_{4}+M_{4} M_{5}^{-1}\right) a R_{s}^{1 / 2}+F_{1} a R^{1 / 2} M_{l}\left(1+M_{5}\right)=0,
\end{aligned}
$$




$$
\begin{aligned}
& A_{1} a R^{1 / 2}\left(1-M_{2}-M_{2} M_{5}\right)-B_{1}\left(\left(\pi^{2}+a^{2}\right)+\operatorname{Pr} \sigma\right)+F_{1} \operatorname{Pr} M_{2} \sigma=0 \\
& A_{1} a R_{S}{ }^{1 / 2} M_{6}+B_{1}\left(\frac{R_{S}}{R}\right)^{\frac{1}{2}} \frac{M_{5}}{M_{6}}\left(\pi^{2}+a^{2}\right) \mathrm{S}_{\mathrm{T}}+C_{1}\left(\tau\left(\pi^{2}+a^{2}\right)+\operatorname{Pr} \sigma\right)=0 \\
& -B_{1} \pi^{2}\left(1-\mathrm{S}_{\mathrm{T}}\right) R_{S}^{\frac{1}{2}}+C_{1} \pi^{2} \frac{M_{5}}{M_{6}} R^{\frac{1}{2}}+F_{1}\left(\pi^{2}+a^{2} M_{3}\right) R_{S}^{\frac{1}{2}}=0
\end{aligned}
$$

For the existence of non-trivial eigen functions the determinant of the coefficients of $A_{1}, B_{1}, C_{l}$, and $F_{1}$ in Eqs (3.3) - (3.6) must vanish. Following the techniques and analysis of Finlayson on Eqs (3.3) - (3.6) leads to

$$
U \sigma^{4}+V \sigma^{3}+W \sigma^{2}+X \sigma+Y=0
$$

where

$$
\begin{aligned}
& U=x_{1} \operatorname{Pr}^{2}, \quad V=c_{1}\left(c_{2}+x_{1}\right) \operatorname{Pr}^{2}+x_{1}^{2} \operatorname{Pr}(1+\tau), \\
& W_{1}=\operatorname{Pr} c_{1}\left(c_{2}+x_{1}\right) x_{1}(1+\tau)+\tau x_{1}^{3}+\operatorname{Ta}^{2} \operatorname{Pr}^{2}+\operatorname{Pr}^{2} c_{1}^{2} c_{2}-a^{2} R_{S} x_{4} M_{6} \operatorname{Pr}, \\
& W_{2}=a^{2}\left[x_{3} x_{2}^{-1} \pi^{2} \operatorname{Pr}\left(\left(1-\mathrm{S}_{\mathrm{T}}\right)+M_{5}\right)-\operatorname{Pr}\left(1+x_{3}\left(1-\mathrm{S}_{\mathrm{T}}\right)\right)\right], \\
& X_{1}=\tau x_{1}^{3} c_{1}\left(c_{2}+x_{1}\right)+x_{1} \operatorname{Pr}(1+\tau)\left(\operatorname{Ta} \pi^{2}+c_{1}^{2} c_{2}\right)-a^{2} R_{S} x_{4}\left(x_{1} \mathrm{~S}_{\mathrm{T}} \frac{M_{5}}{M_{6}}+M_{6}\left(\operatorname{Pr} c_{1}+x_{1}\right)\right) \\
& X_{2}=a^{2}\left[x_{2}{ }^{-1} x_{3} \pi^{2}\left(x_{1} \mathrm{~S}_{\mathrm{T}} \frac{M_{5}^{2}}{M_{6}{ }^{2}}+\left(\tau x_{1}+\operatorname{Pr} c_{1}\right)\left(1-\mathrm{S}_{\mathrm{T}}\right)+M_{5}\left(x_{1}+\operatorname{Pr} c_{1}\right)\right)+\right. \\
& \left.-\left(x_{1} \tau+\operatorname{Pr} c_{1}\right)\left(1+x_{3}\left(1-\mathrm{S}_{\mathrm{T}}\right)\right)\right] \text {, } \\
& Y_{1}=x_{1}^{2} \tau\left(\operatorname{Ta} \pi^{2}+c_{1}^{2} c_{2}\right)-a^{2} R_{S} x_{4} c_{1} x_{1}\left(\mathrm{~S}_{\mathrm{T}} \frac{M_{5}}{M_{6}}+M_{6}\right), \\
& Y_{2}=a^{2}\left[x_{2}^{-1} x_{3} \pi^{2} x_{1} c_{1}\left(\mathrm{~S}_{\mathrm{T}} \frac{M_{5}^{2}}{M_{6}^{2}}+\tau\left(1-\mathrm{S}_{\mathrm{T}}\right)+M_{5}\right)-\tau c_{1} x_{1}\left(1+x_{3}\left(1-\mathrm{S}_{\mathrm{T}}\right)\right)\right]
\end{aligned}
$$

$W=W_{1}+W_{2} R, X=X_{I}+X_{2} R, Y=Y_{1}+Y_{2} R$, where $R$ is the thermal critical Rayleigh number. Also,

$$
\begin{array}{lll}
x_{1}=\pi^{2}+a^{2}, & x_{2}=\pi^{2}+a^{2} M_{3}, & x_{3}=M_{1}\left(1+M_{5}\right), \\
x_{4}=\left(1+M_{4}+\frac{M_{4}}{M_{5}}\right), & c_{1}=\frac{1}{k}+x_{1}, & c_{2}=\delta+x_{1} .
\end{array}
$$


For obtaining stationary instability the time independent term is made equal to zero. The critical thermal Rayleigh number for stationary instability is obtained from Eq.(3.8), using the formula

$$
R_{S C}=\frac{N r}{D r}
$$

where

$$
\begin{aligned}
& N r=\left(\frac{\mathrm{Ta} \pi^{2} x_{1}}{c_{1}}+x_{1} c_{1} c_{2}\right)-a^{2} R_{S} x_{4}\left(\mathrm{~S}_{\mathrm{T}} \frac{M_{5}}{M_{6}}+M_{6}\right) \tau^{-1}, \\
& D r=a^{2}\left[\left(1+x_{3}\left(1-\mathrm{S}_{\mathrm{T}}\right)\right)-x_{2}^{-1} x_{3} \pi^{2} \tau^{-1}\left(\mathrm{~S}_{\mathrm{T}} \frac{M_{5}^{2}}{M_{6}^{2}}+\tau\left(1-\mathrm{S}_{\mathrm{T}}\right)+M_{5}\right)\right] .
\end{aligned}
$$

It is worthwhile to mention that in the absence of the magnetic field dependent viscosity, the Taylor number and the permeability of the porous medium, the critical thermal Rayleigh number given by Eq.(3.8), reduces to the critical thermal Rayleigh number obtained by Vaidyanathan et al. (2005). In the absence of the field dependent viscosity and the Soret parameter this Eq.(3.8) reduces to the thermal Rayleigh number obtained by Vaidyanathan et al. (1997). For very large $M_{1}$, one gets the results for the magnetic mechanism, and the critical thermomagnetic Rayleigh number for stationary mode is obtained using

$$
N_{S C}=R M_{1}=\frac{N r}{D r}
$$

where

$$
\begin{aligned}
& N r=\left(\frac{\mathrm{Ta}^{2} x_{1}}{c_{1}}+x_{1} c_{1} c_{2}\right)-a^{2} R_{S} x_{4}\left(\mathrm{~S}_{\mathrm{T}} \frac{M_{5}}{M_{6}}+M_{6}\right) \tau^{-1}, \\
& D r=a^{2}\left(1+M_{5}\right)\left[\left(1-\mathrm{S}_{\mathrm{T}}\right)-\frac{\pi^{2} \tau^{-1}\left(\mathrm{~S}_{\mathrm{T}} \frac{M_{5}^{2}}{M_{6}^{2}}+\tau\left(1-\mathrm{S}_{\mathrm{T}}\right)+M_{5}\right)}{x_{2}}\right] .
\end{aligned}
$$

The conditions for the onset of oscillatory stabilities are obtained as follows. Taking $\sigma=i \sigma_{l}$ and $\sigma_{l}^{2}>0$ in (28) we get

$$
R_{0 S C}=\frac{N r}{D r}
$$

where,

$$
R_{0 S C}=\frac{U W_{2} \sigma_{1}^{4}+\left(V X_{2}-W_{1} W_{2}\right) \sigma_{1}^{2}-X_{1} X_{2}}{X_{2}^{2}+\sigma_{1}^{2} W_{2}^{2}} \quad \text { and } \quad \sigma_{1}^{2}=\frac{\left(X_{2} W_{1}-X_{1} W_{2}\right)}{X_{2} U-V W_{2}}
$$


where if $R_{0 C}>R_{S C}$, then the system stabilizes through stationary mode. If $R_{0 C}<R_{S C}$, then the system stabilizes through oscillatory mode, where $R_{O C}$ and $R_{S C}$ are the critical thermal Rayleigh numbers for the oscillatory and stationary convection system.

\section{Results and discussions}

The Soret-driven convective instability of a two component ferrofluid in the presence of acmagnetic field dependent viscosity in a rotating sparsely distributed porous medium has been analyzed. A Brinkman model is used. A Linear stability analysis has been carried out as the perturbations are assumed to be small. The magnetization parameter $M_{l}$ is taken to be 1000 . The Soret parameter $\mathrm{S}_{\mathrm{T}}$ is allowed to vary from -0.002 to 0.002 . The salinity Rayleigh number $R_{S}$ is assumed to take values from -500 to 500 and the magnetization parameter $M_{3}$ is varied from 5 to 25 . The ratio of mass transport to heat transport $\tau$ is assumed to have values from 0.05 to 0.13 . The Prandtl number Pr is assumed to be 0.01 . For these fluids $M_{2}$ will have a negligible value and hence is taken as zero. The magnetic field dependent viscosity $\delta$ is allowed to take values from 0.01 to 0.07 . The Talyor number Ta which decides about the amount of rotation is allowed to vary from 10 to $10^{8}$. The permeability of the porous medium $k$ is given values ranging from 0.1 to 0.9 .

Figure 1 represents the variation of the critical thermal Rayleigh number $R_{c}$ versus the permeability of the porous medium $k$ for different values of $\delta$, the dependent viscosity. It is seen from the figure that the system destabilizes as the permeability of the porous medium $k$ increases. This is indicated by a decrease in $R_{c}$ values. The reason is that as the pore size increases, it becomes easier for the flow to destabilize the system. It is observed from the figure that the dependent viscosity $\delta$ is found to stabilize the system.

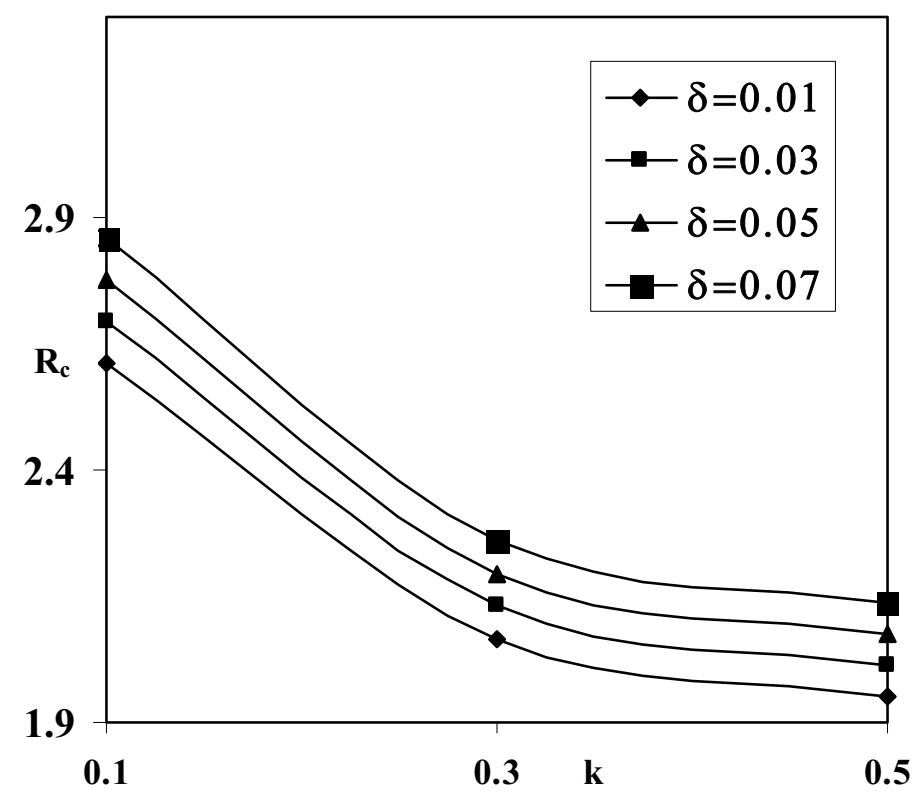

Fig.1. Variation of $R_{c}$ versus $k$ for different values of $\delta$.

From Fig.2, the critical thermal Rayleigh number variation with respect to the magnetization parameter $M_{3}$ for different values of the permeability of the porous medium $k$ indicates that the system destabilizes as the magnetization parameter $M_{3}$ increases. This is seen by a decrease in $R_{c}$ values. A destabilizing trend of $k$ is also seen in this figure. 


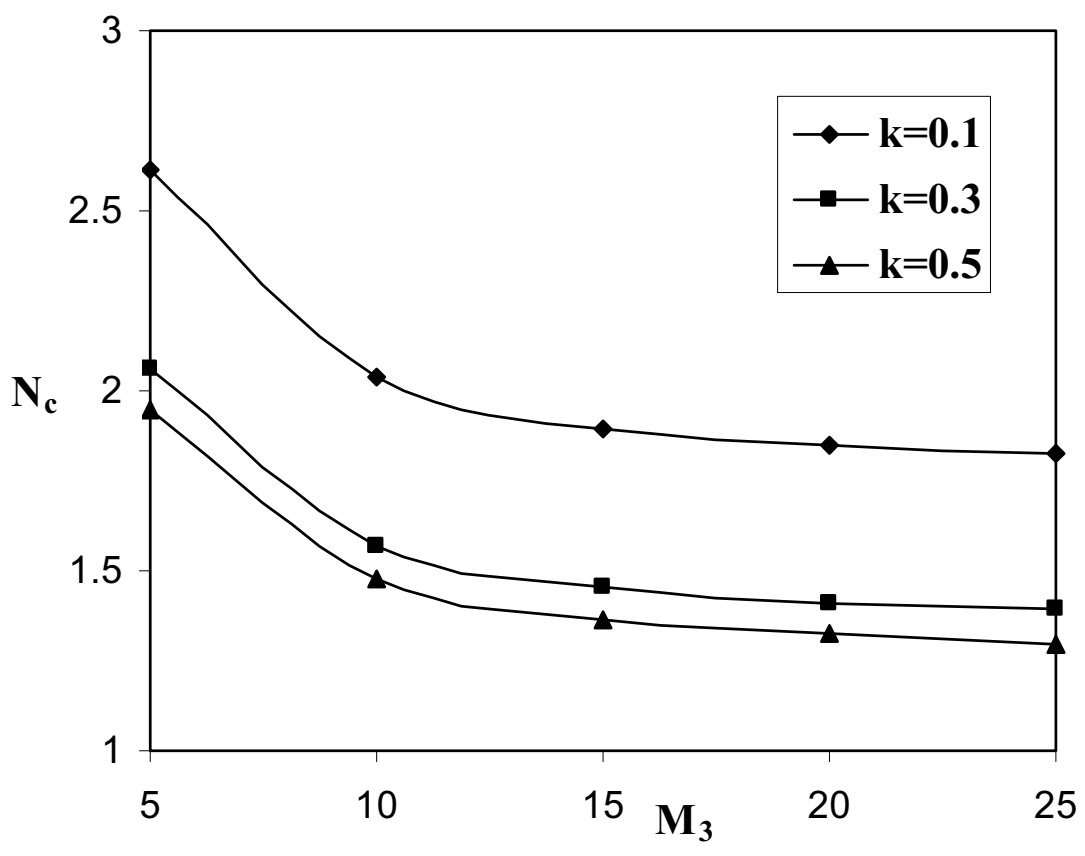

Fig.2. Variation of $R_{c}$ versus $M_{3}$ for different values of $k$.

It is observed from Figs $3 \mathrm{a}, 3 \mathrm{~b}$ and $3 \mathrm{c}$ that the Soret parameter $\mathrm{S}_{\mathrm{T}}$ stabilizes the system, thereby delaying the onset of convection. All the three graphs exhibit a stabilizing trend. This is due to the fact that the modulation of the salinity gradient by the temperature gradient promotes stabilization. Positive values of $\mathrm{S}_{\mathrm{T}}$ stabilize the system more. The destabilizing trend of $M_{3}$ and $k$ is also seen from Figs $3 \mathrm{a}$ and $3 \mathrm{~b}$. A stabilizing behaviour of $\delta$ is seen from Fig.3c.

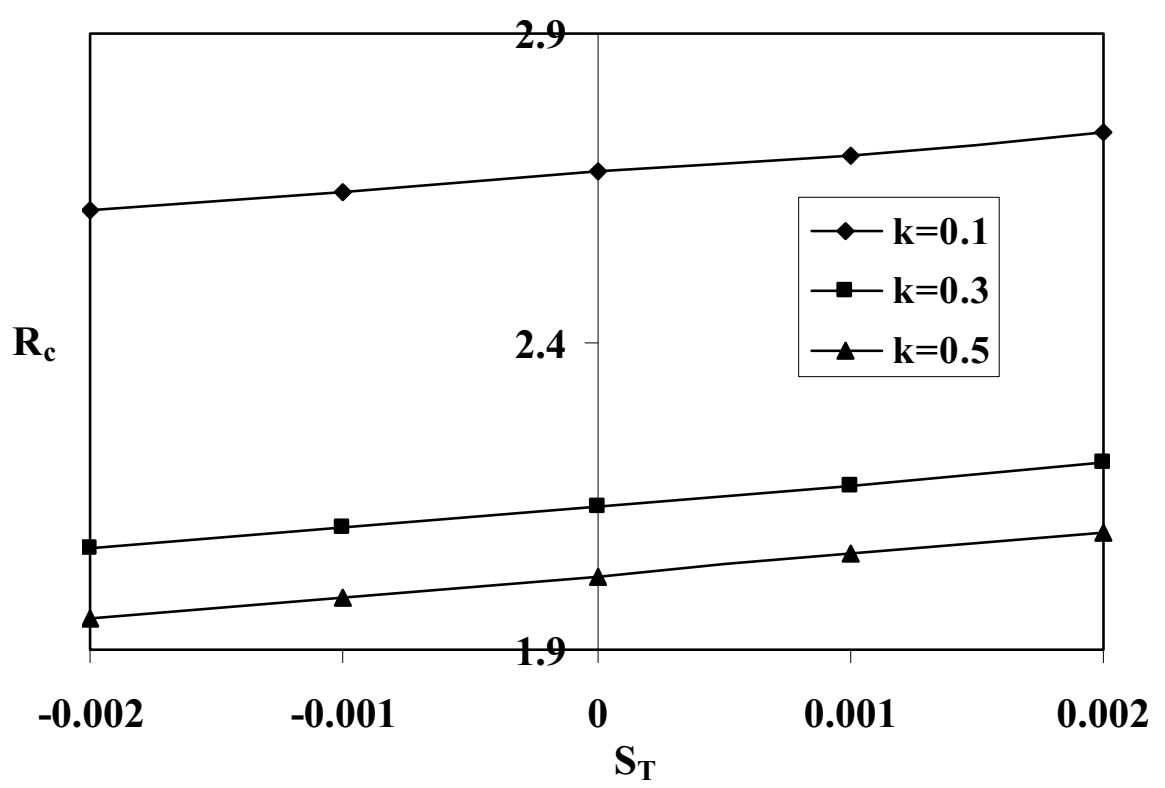

Fig.3a. Variation of $R_{c}$ versus $\mathrm{S}_{\mathrm{T}}$ for different values of $k$. 


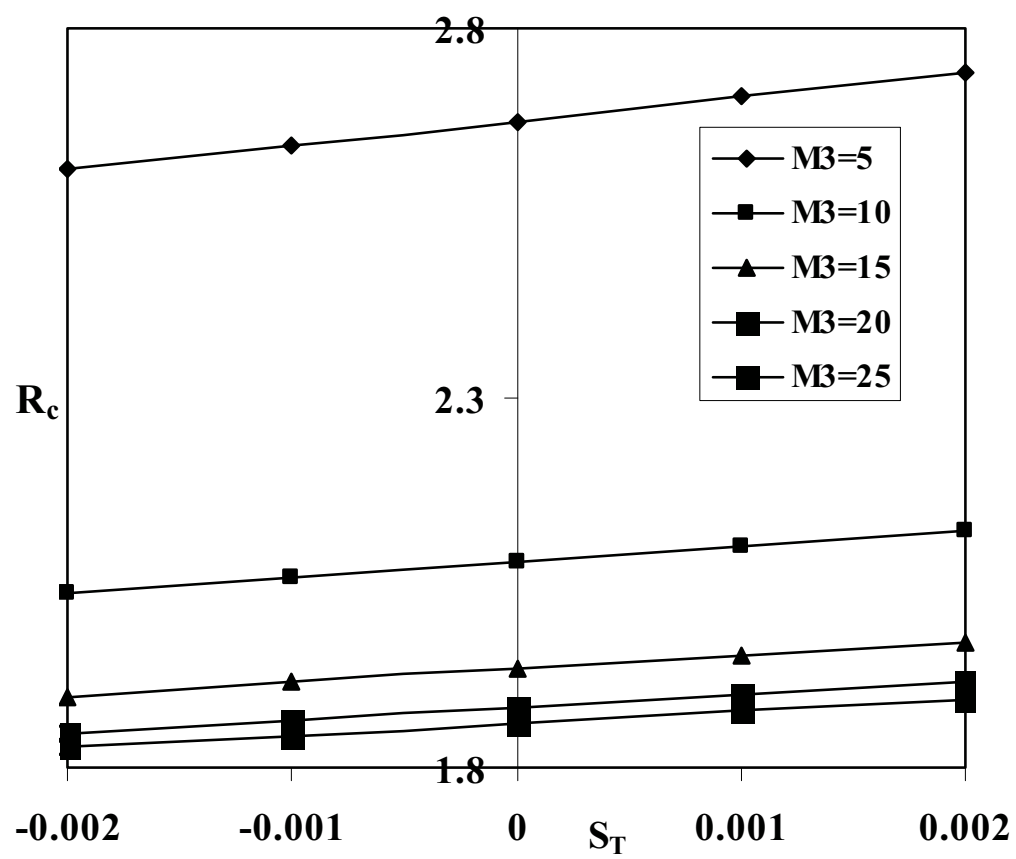

Fig.3b. Variation of $R_{c}$ versus $\mathrm{S}_{\mathrm{T}}$ for different values of $M_{3}$.

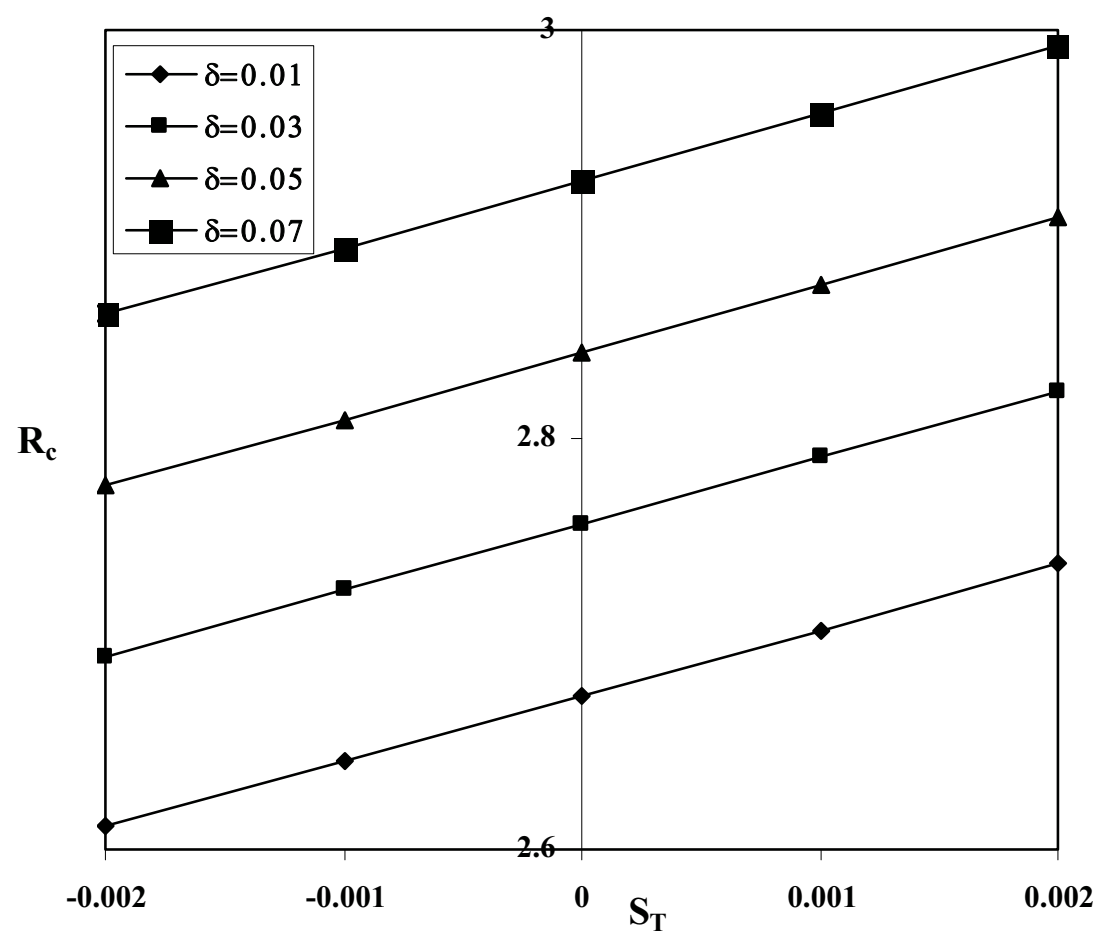

Fig.3c. Variation of $R_{c}$ versus $\mathrm{S}_{\mathrm{T}}$ for different values of $\delta$.

Figures $4 \mathrm{a}, 4 \mathrm{~b}$ and $4 \mathrm{c}$ analyze the variation of $R_{C}$ versus LogTa for different values of $M_{3}, k$ and $\delta$. When LogTa increases from 1 to 5 , the stabilization is not much pronounced. But when it takes values from 6 to 8 , the increase in $R_{c}$ values is maximum. 
Figures $5 \mathrm{a}$ and $5 \mathrm{~b}$ investigate the variation of $R_{c}$ versus $M_{3}$ for different values of $\delta$ and $R_{c}$ versus $\delta$ for different values of $M_{3}$. Both figures illustrate that as $M_{3}$ increases, the values of $R_{c}$ decrease for small values of $\delta$, whereas for higher values of $\delta, R_{c}$ decreases for lower values of $M_{3}$, and then increases for higher values of $M_{3}$.

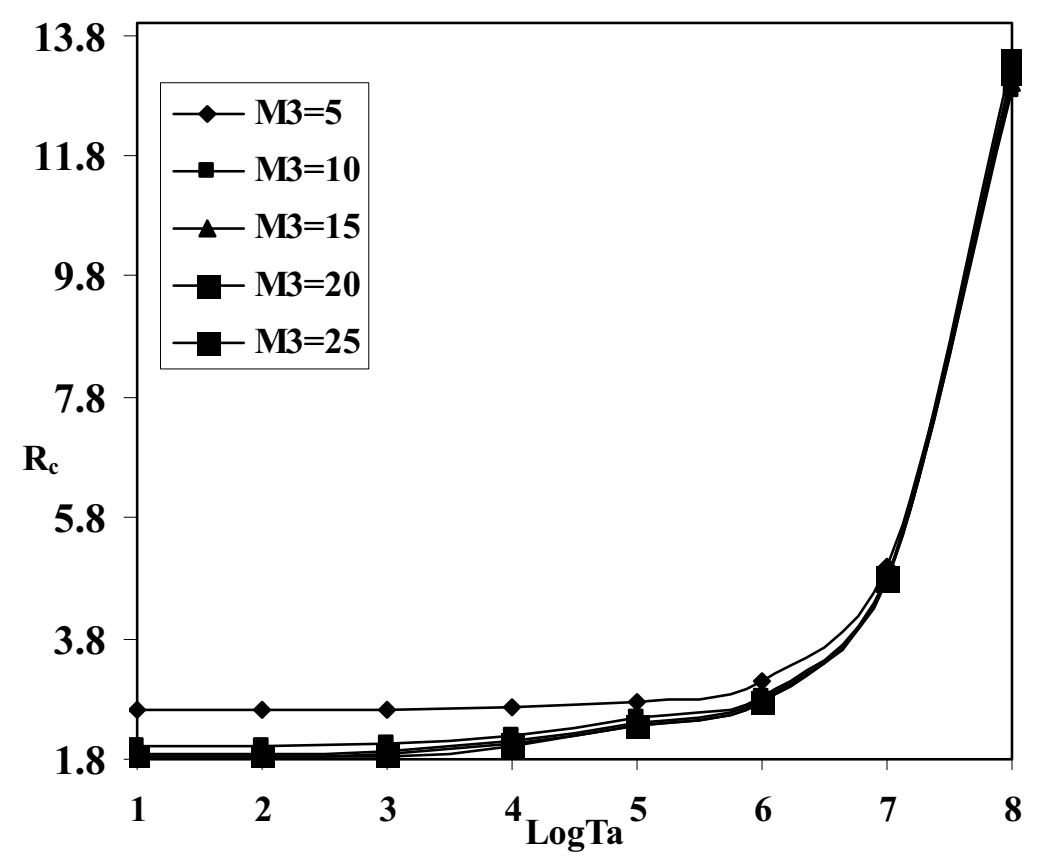

Fig.4a. Variation of $R_{c}$ versus $\log T a$ for different values of $M_{.3}$ when $\operatorname{Pr}=0.01$.

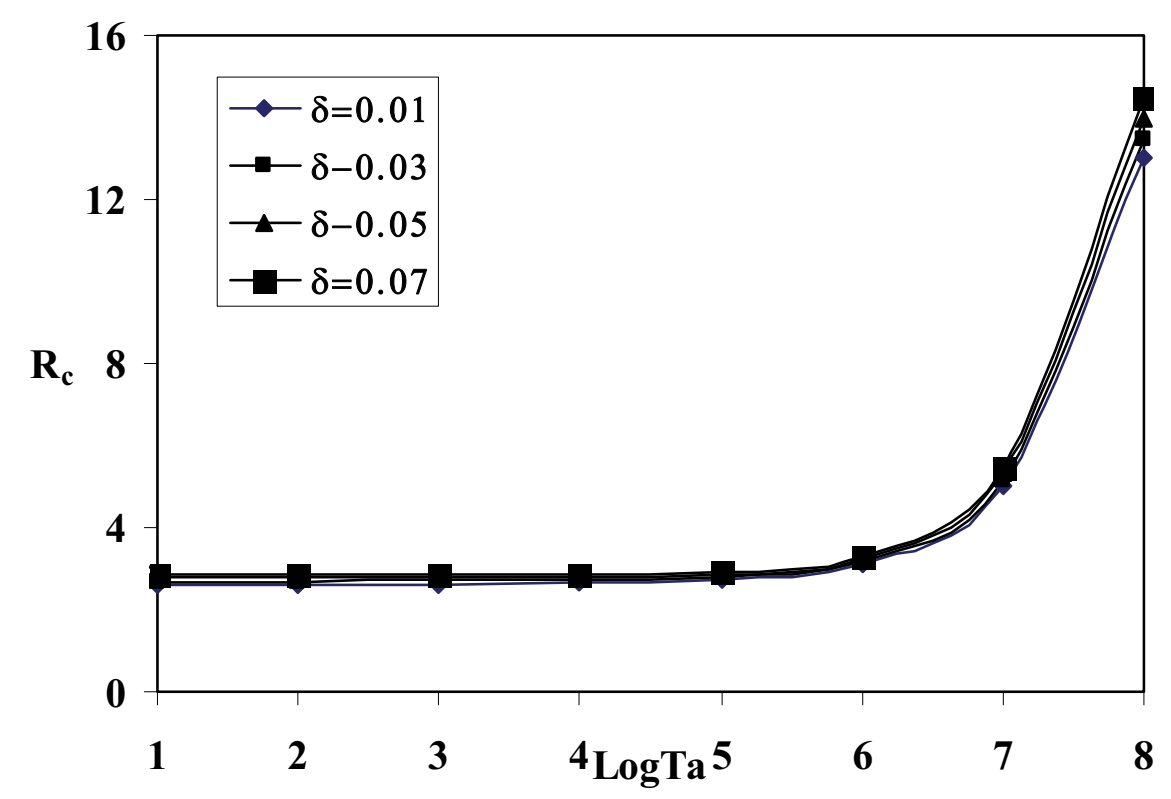

Fig.4b. Variation of $R_{c}$ versus LogTa for different values of $\delta$. 


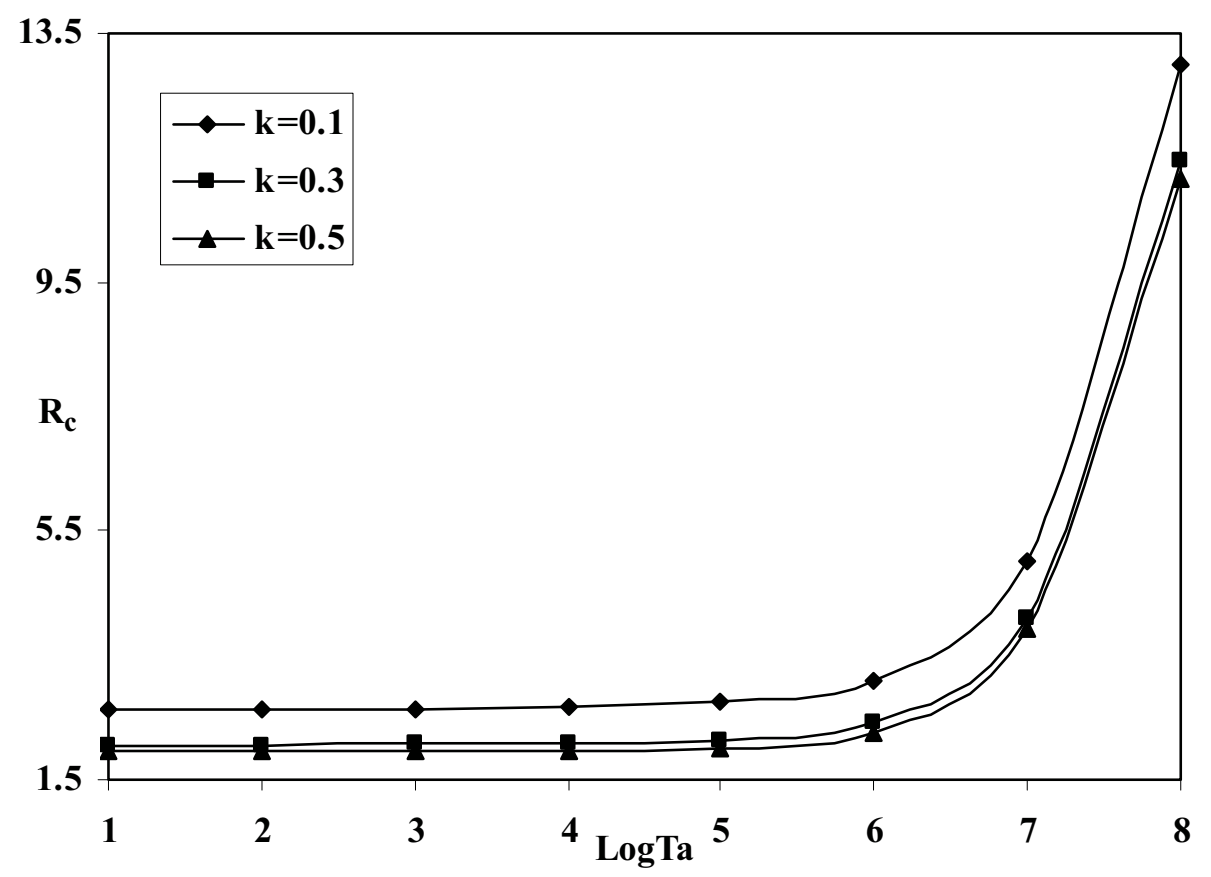

Fig.4c. Variation of $R_{c}$ versus $\log$ Ta for different values of $k$.

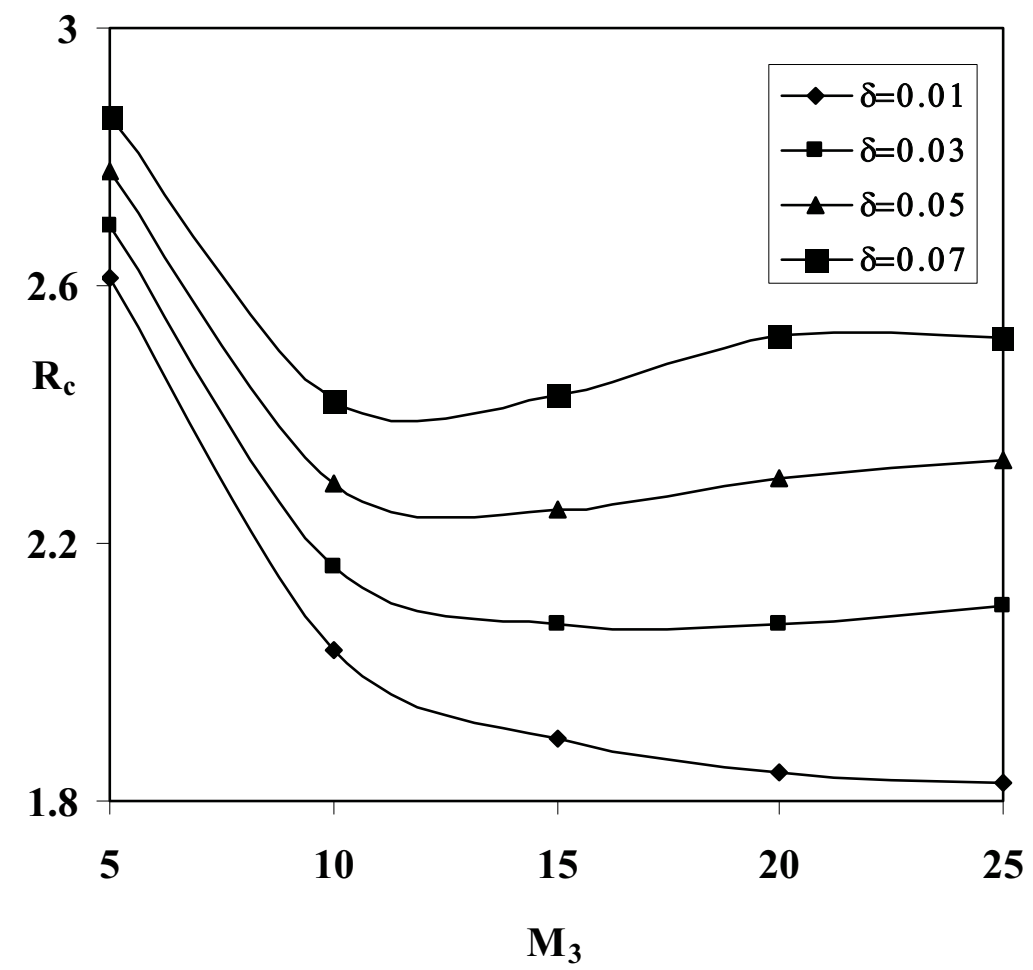

Fig.5a. Variation of $R_{c}$ versus $M_{3}$ for different values of $\delta$. 


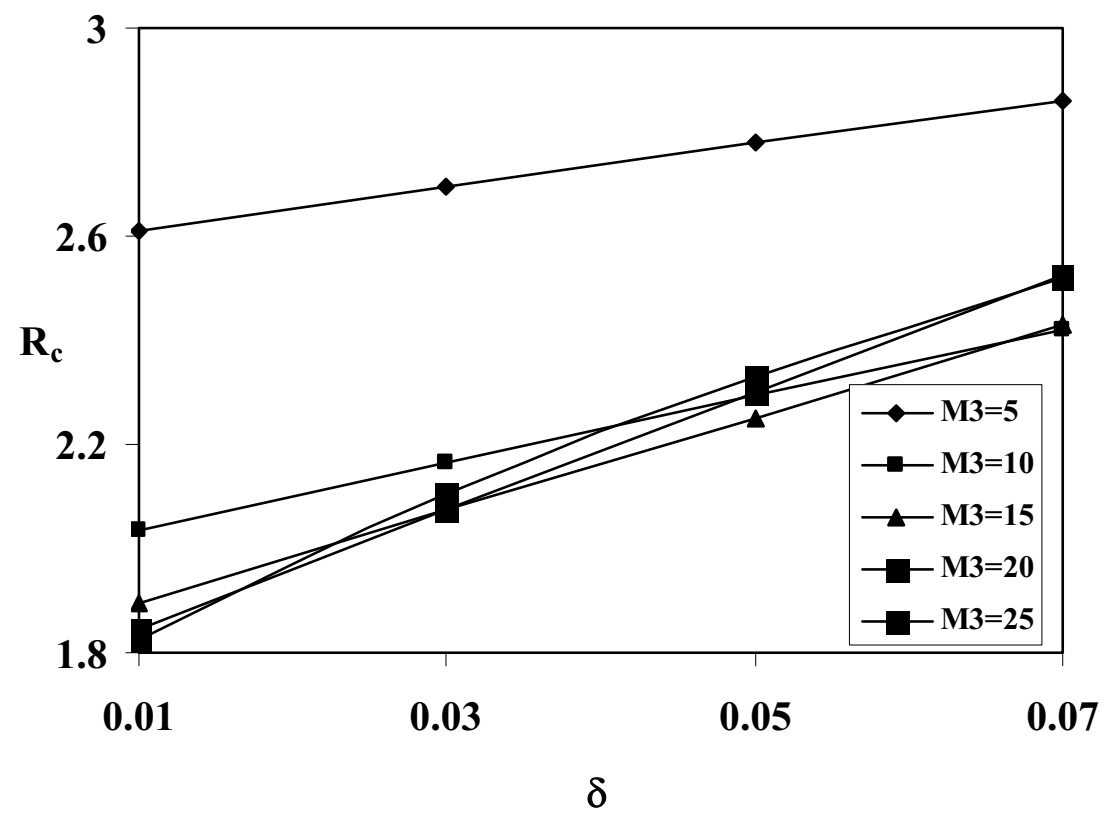

Fig.5b. Variation of $R_{c}$ versus $\delta$ for different values of $M_{3.3}$.

Figure 6 analyzes the variation of $R_{c}$ versus $\delta$ for different values of Ta. The figure exhibits a stabilizing behaviour. The stabilization is minimal when the Taylor number Ta assumes values from 10 to $10^{5}$, and then it increases phenomenally. This is indicated by an increase in $R_{c}$ values.

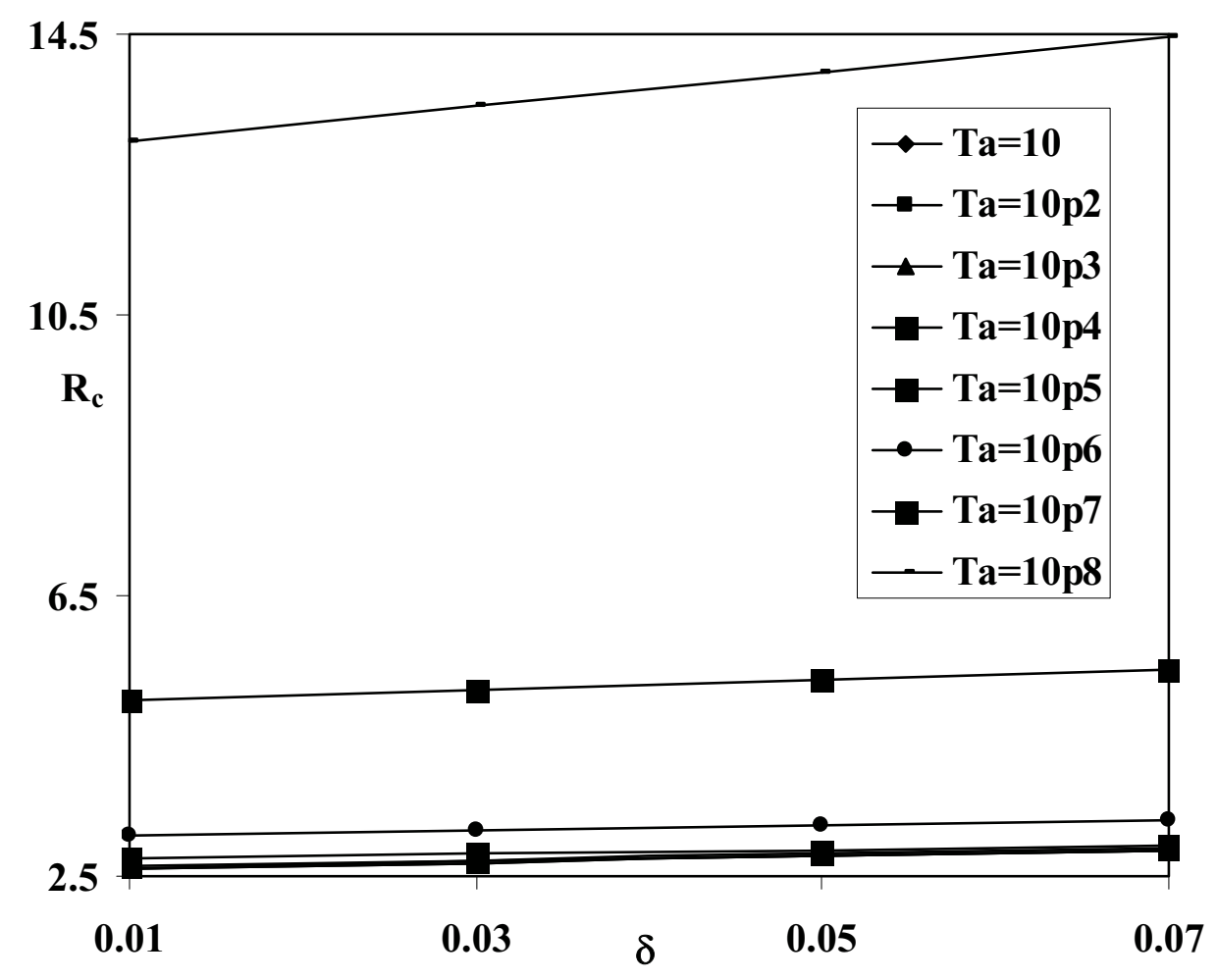

Fig.6. Variation of $R_{c}$ versus $\delta$ or different values of Ta.

In this figures $10 p n$ indicates 10 to the power of $n$, where $n=2,3,4,5,6,7,8$. 


\section{Nomenclature}

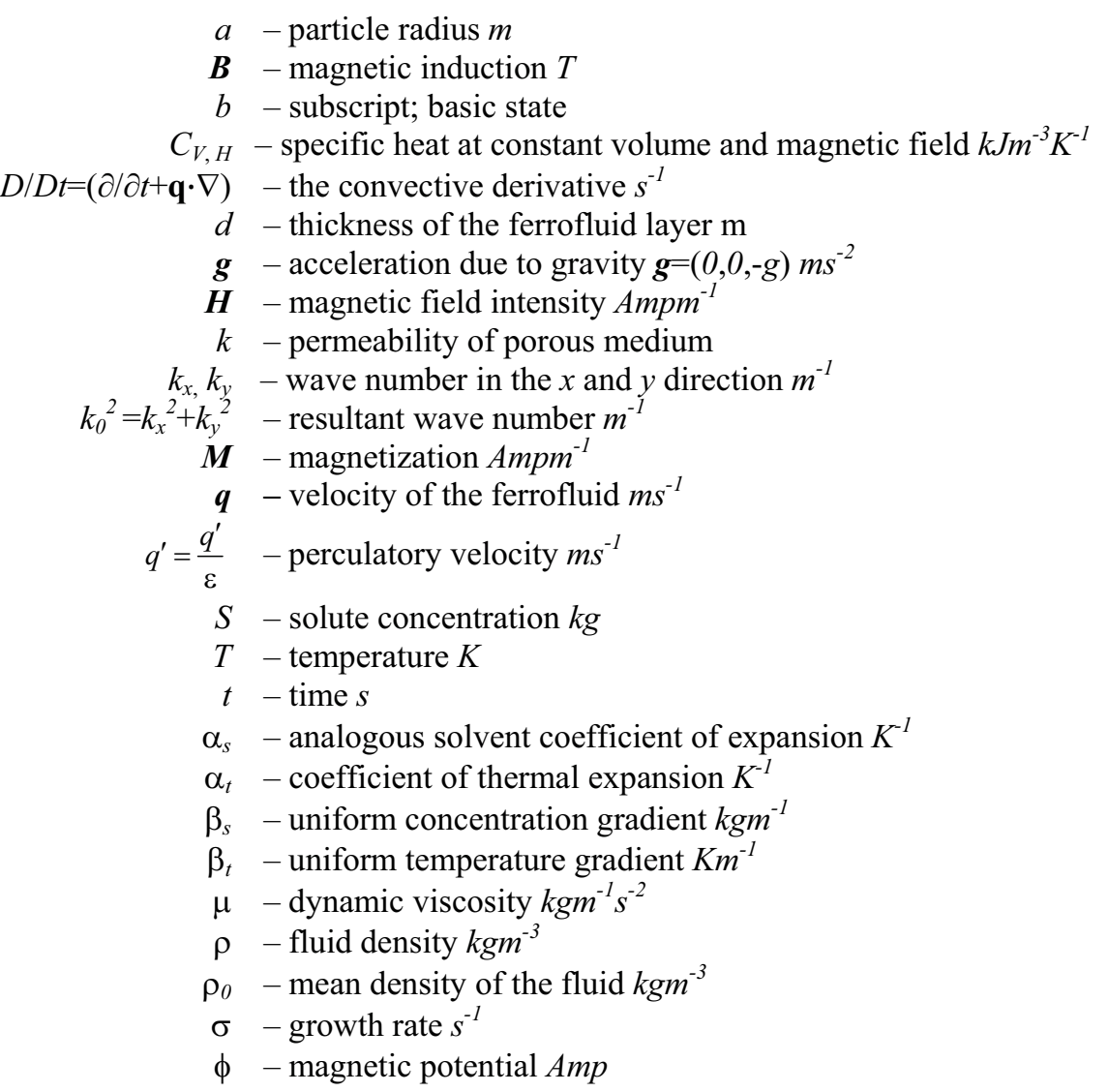

\section{References}

Chandrasekhar S. (1961): Hydrodynamic and Hydromagnetic Stability. - London: Oxford University Press.

Charier-Mojtabi M.C., Elhajjar B. and Mojtabi A. (2007): Analytical and numerical stability analysis of Soret driven convection in a horizontal porous layer. - Phys. of Fluids, vol.19, pp.124104-13.

Cowley M.D and Rosensweig R.E. (1967): The interfacial stability of a ferromagnetic fluid. - J. Fluid Mech., vol.30, pp.671-688.

Finlayson B.A. (1970): Convective instability of ferromagnetic fluids. - J. Fluid. Mech., vol.40, pp.753-767.

Hemalatha R. and Sivapraba N. (2012): Effect of magnetic field dependent viscosity on ferroconvection in a sparsely distributed anisotropic porous medium in the presence of horizontal thermal gradient. - Ind. J. Pure. Appl. Phys., vol.50, pp.907-914.

Hemalatha R., Sekar R. and Vaidyanathan G. (2012 ): Effect of dust particles on a Soret- driven ferro thermohaline convection in a rotating medium. - Int. J. of Appl., Mech. and Engng., vol.17, No.2, pp.367-381.

Hemalatha R., Sekar R. and Vaidyanathan G. (2011): Effect of rotation on a Soret- driven thermohaline convection in dusty ferrofluids saturating a porous medium. - Int. J. of Appl., Mech. and Engng., vol.16, No.4, pp.1021-1036.

Hupper H.E and Turner J.S. (1981): Double diffusive convection. - J. Fluid Mech., vol.106, pp.299-329.

Hurle D.T.J. and Jakemann E.J. (1971): Soret-driven thermo solutal convection. - Fluid Mech., vol.47, pp.667-687.

Kim M.C. (2011): Linear stability analysis on the onset of Soret driven motion in nanoparticles suspension. - The European Physical Journal, vol.34, pp.27. 
Kushal S., Paras R. and Kushal S. (2011b): Revolving ferrofluid flow under the influence of magnetic field dependent viscosity and porosity with rotating disk on. - J. Electro Magn. Analysis and Applications (Scientific Research), vol.3(9), pp.378-386.

Kushal S., Paras R., Anupam B. and Kushal S. (2010): Effect of magnetic field dependent viscosity on revolving ferrofluid. - J. Magn. Magn. Mater., vol.322, No.21, pp.3476-3480.

Kushal S., Paras R., Kushal S. and Anupam B. (2011a): Effect of porosity on revolving ferrofluid with rotating diskInt. J. Fluids Engng., vol.3, No.3, pp.261-271.

Lalas D.P. and Carmi S. (1971): Thermo convective stability of ferrofluids. - Phys Fluids, vol.14, No.2, pp.436-437.

Lange A. (2004): Thermomagnetic convection of magnetic fluids in a cylindrical geometry. - Phys. Rev., E.70, pp.046308.

Ramanathan and Muchikel N. (2006): Effect of temperature-dependent viscosity on ferroconvection in a porous medium. - Int. J. of Appl. Mech. and Engng., vol.11, No.1, pp.93-104.

Rosensweig R.E. (1985): Ferrohydrodynamics. - Cambridge: Cambridge University Press.

Schechter R.S and Velarde M. (1974): The component Benard problem - Adv. in Phys., vol.26, pp.265-301.

Schwab L., Hilderbrandt U. and Stierstadt K. (1983): Magnetic Benard convection. - J. Magn. Magn. Mater., vol.39, pp.113-114.

Sekar R., Vaidyanathan G. and Hemalatha R. (2008): Soret-driven thermohaline convection in dusty ferrofluids saturating a porous medium. - Int. J. of Appl., Mech. and Engng., vol.13, No.4, pp.1003-1018.

Sekar R., Vaidyanathan G. and Hemalatha R. (2009): Effect of presence of dust particles on Soret-driven ferrothermohaline convection. - Int. J. of. Appl. Mech. and Engng., vol.14, No.2, pp.509-522.

Sekar R., Vaidyanathan G. and Ramanathan A. (1998): Effect of rotation on ferrothermohaline convection saturating a porous medium. - Int. J. Eng. Sci., vol.5, pp.445-452.

Sekar R., Vaidyanathan G. and Ramanathan A. (2000): Effect of rotation on ferrothermohaline convection. - J. Magn. Magn. Mater., vol.218, pp.266-272.

Sekar R., Vaidyanathan G., Hemalatha R. and Sendhilnathan S. (2006): Effect of sparse distribution pores in a Soret driven ferrothermohaline convection. - J. Magn. Magn. Mater., vol.302, pp.20-28.

Sekar R., Vaidyanathan G., Hemalatha R. and Sendhilnathan S. (2007): Effect of Coriolis force on Soret driven thermohaline convective system. - Int. J. Mathematical Sci., vol.6, No.3-4, pp.666-673.

Sekar R., Vaidyanathan G. and Ramanathan A. (1993): The ferroconvection in fluids saturating a rotating densely packed porous medium. - Int. J. Eng. Sci., vol.31, No.2, pp.241.

Shevtsova M., Melnikov D.E. and Clegros J. (2006): Onset of convection in Soret driven instability. - Phys. Rev., E.73, p.047302.

Shliomis M.I. and Souhar M. (2000): Self-oscillatory convection caused by Soret-effect. - Europhys Lett., vol.49, No.1, pp.55.

Sunil A. and Sharma R.C. (2005a): Effect of magnetic field dependent viscosity on thermosolutal convection in ferromagnetic fluid. - App. Math. Comp., vol.163, No.3, pp.1197-1214.

Sunil A., Poonam S. and Amit M. (2011): A non linear stability analysis of a rotating double diffusive magnetized ferrofluid. - Appl. Maths. Computation., vol.218, No.6, pp.2785-2799.

Sunil D. and Sharma R.C. (2005b): Effect of magnetic field dependent viscosity on thermosolutal convection in ferromagnetic fluid saturating a porous medium. - Trans. Porous Media, vol.60, No.3, pp.251-274.

Turner J.S. (1974): Double diffusive phenomena. - Ann. Rev Fluid Mech., vol.6, pp.37.

Vaidyanathan G and Sekar R (2002b): Effect of magnetic field dependent viscosity on ferroconvection in rotating medium. - Ind. J. Pure. Appl. Phys., vol.40, pp.159-165.

Vaidyanathan G., Sekar R., Vasanthakumari R. and Ramanathan A. (2002c): Effect of magnetic field dependent viscosity on ferroconvection in a rotating sparsely distributed porous medium. - J. Magn. Magn. Mater., vol.250, pp.65-76. 
Vaidyanathan G., Sekar R. and Ramanathan A. (1995): Ferrothermohaline convection in a porous medium. - J. Magn. Magn. Mater., vol.176, pp.321-330.

Vaidyanathan G., Sekar R. and Ramanathan A. (1997): Ferrothermohaline convection.- J. Magn. Magn. Mater., vol.176, pp.321-330.

Vaidyanathan G., Sekar R., Hemalatha R., Vasanthakumari R. and Sendhilnathan S. (2005): Soret driven ferrothermohaline convection. - J. Magn. Magn. Mater., vol.288.

Vaidyanathan G., Ramanathan A. and Maruthamanikandan S. (2002a): Effect of magnetic field dependent viscosity on ferroconvection in sparsely distributed porous medium. - Ind. J. Pure. Appl. Phys., vol.40, pp.166-171.

Voelker T. and Odenbach S. (2005): Thermo diffusion in ferrofluids in the presence of a magnetic field. - Phys Fluids., vol.17, pp.037104.

Received: August 11, 2013

Revised: November 12, 2013 